\title{
Application of Photoacoustic Calorimetry to the Photofragmentation of $\mathrm{Cr}(\mathrm{CO})_{6}$
}

\author{
JOHN D. SIMON, MARK BERNSTEIN and KEVIN S. PETERS $\dagger$ \\ Contribution from the Department of Chemistry, Harvard University, Cambridge, \\ Massachusetts, 02138
}

Photolysis of $\mathrm{Cr}(\mathrm{CO})_{6}$ in cyclohexane yields solvated pentacarbonyl species within 25 ps. Using high-speed photoacoustic calorimetry, we have determined the heat of reaction for this photofragmentation, directly measuring the energy required to break a single metal-carbonyl bond followed by solvation of the fragments. The energy required to photochemically dissociate a single $\mathrm{CO}$ in cyclohexane exceeds the gas phase average bond dissociation energy by at least $11 \mathrm{kcal} / \mathrm{mol}$. However, the determined heat of reaction agrees well with the activation energies derived from thermal decomposition kinetics.

The primary photochemical reaction of electronically excited chromium hexacarbonyl in condensed phase is the dissociation of a single carbonyl ligand, ${ }^{1}$ Eq. (1).

$$
\mathrm{Cr}(\mathrm{CO})_{6} \longrightarrow \mathrm{Cr}(\mathrm{CO})_{5}+\mathrm{CO}
$$

Time-resolved picosecond absorption studies ${ }^{2}$ have shown that a pentacarbonyl species appears within 25 ps of photolysis. The absorption spectrum of this intermediate is solvent-dependent. Mixed solvent studies suggest that the transient species acquires a coordinated solvent molecule to occupy the site vacated by the dissociated carbonyl.

$$
\mathrm{Cr}(\mathrm{CO})_{6} \longrightarrow \mathrm{Cr}(\mathrm{CO})_{5} \mathrm{~S}+\mathrm{CO}
$$

Although the spectroscopy and photochemistry of these intermediates have been intensively studied, ${ }^{1}$ the thermodynamic properties of these reactions are poorly understood. 
In the gas phase, the photofragmentation of $\mathrm{Cr}(\mathrm{CO})_{6}$ proceeds rapidly to completion, ${ }^{3}$ and the only observable photoproducts are $\mathrm{CO}$ and bare metal atoms. As a result, gas phase studies can only determine the average strength of all six metal-carbonyl bonds. ${ }^{4}$ Solution photolysis of $\mathrm{Cr}(\mathrm{CO})_{6}$, on the other hand, produces pentacarbonyl derivatives in high yield. Thus a determination of the $\mathrm{M}-\mathrm{CO}$ bond strength is essential to the civvelopment of a complete picture of the reactions of metal carbonyl in condensed media. In particular, stabilization of the resulting unsaturated coordination compound by solvent molecules might influence the overall utility of a given substitution reaction. In order to examine these effects we have applied photoacoustic calorimetry to determine the heat of reaction for Eq.

(2) in cyclohexane.

\section{EXPERIMENTAL}

The application of photoacoustic spectroscopy to determine quantum yields for heat deposition has been developed by Rockley, ${ }^{5}$ Kirkbright, ${ }^{6}$ Starobogatov, ${ }^{7}$ and others, primarily in order to obtain precise fluorescence quantum yields. ${ }^{8}$ Previous studies have been limited, however, to investigations of stable species.

By using a pulsed laser source and a high-speed piezoelectric detector, we have demonstrated the feasibility of photocalorimetric studies of short-lived, transient molecules with lifetimes on the order of microseconds or less. ${ }^{9}$ A complete treatment of this technique will appear elsewhere $;^{9}$ in the present paper we include a qualitative description of the method and a summary of the experimental procedure.

Photolysis is initiated by a 25 ps pulse of $355 \mathrm{~nm}$ UV light generated by frequency-tripling the output of a Nd:YAG laser (Quantel YG400). Each pulse is attenuated by absorbing filters and is individually measured by a pyrolectric probe (Laser Precision Rj-7200/RjP-735). Typical pulses have energies of a few hundred microjoules.

Pulses are weakly focussed to a spot size of a few millimeters and are then directed into a $1 \mathrm{~cm}$ quartz sample cuvette. Photoacoustic signals are detected by a piezoelectric microphone ${ }^{10}$ clamped onto the side of the cuvette, amplified, ${ }^{11}$ gated and integrated by an Evans Associates integrator (Model 4130), and recorded by a microcom- 
puter-based controller. ${ }^{12}$ The energy and the photoacoustic amplitude are recorded for each laser pulse, and averaged over eighty pulses to reduce background noise and fluctuations. The microcomputer controller is programmed to ignore pulses whose energies do not fall within a specified range, permitting us to reject anomalous laser pulses.

The linear relationship between heat deposition and photoacoustic amplitude is well understood. ${ }^{5-9}$ Provided the exciting pulse is not so intense as to induce non-linear effects (e.g., sample bleaching or multiphoton absorption), we observe that photoacoustic amplitudes are directly proportional to individual pulse energies. By varying sample concentration we obtain the photoacoustic equivalent of Beer's law, finding the photoacoustic signal to scale linearly with sample concentration.

$$
|V|=K \Phi_{n r} E_{p} A
$$

Here, $|V|$ is the observed photoacoustic amplitude, $E_{p}$ the UV pulse energy, $A$, the sample absorbance, $\Phi_{n r}$ the sample chromophore's quantum yield for heat deposition, ${ }^{13}$ and the proportionality constant $K$ is determined by the instrumental sensitivity.

The magnitude of the observed photoacoustic signal clearly depends both the light energy absorbed and on the efficiency $\Phi_{n r}$ with which absorbed electromagnetic energy is converted to heat (the quantum yield for non-radiative processes). If excitation triggers endothermic chemical changes, a fixed fraction of the absorbed energy will be consumed by these chemical process, and cannot appear as heat. The observed photoacoustic signal will be reduced in magnitude.

The photoacoustic calorimeter was calibrated as previously described. ${ }^{9}$ In Figure 1, we plot the estimated non-radiative quantum yield against the observed photoacoustic amplitudes for the standardization compounds in cyclohexane solutions (parameters used in estimating $\Phi_{n r}$ are given in Table I). The calibration of EtOH has been reported previously. ${ }^{9}$ In light of the considerable uncertainties inherent in the quantum-yield measurements upon which the calibration curve is based, the correlation between predicted $\Phi_{n r}$ and measured amplitudes is quite satisfactory.

Having determined this calibration, we can estimate the quantum yield for heat deposition of any molecule by determining the slope $|V| / E_{p} A$ of the plot of observed signal versus sample absorbance. Note, however, that the calibration curve applies only to a single 


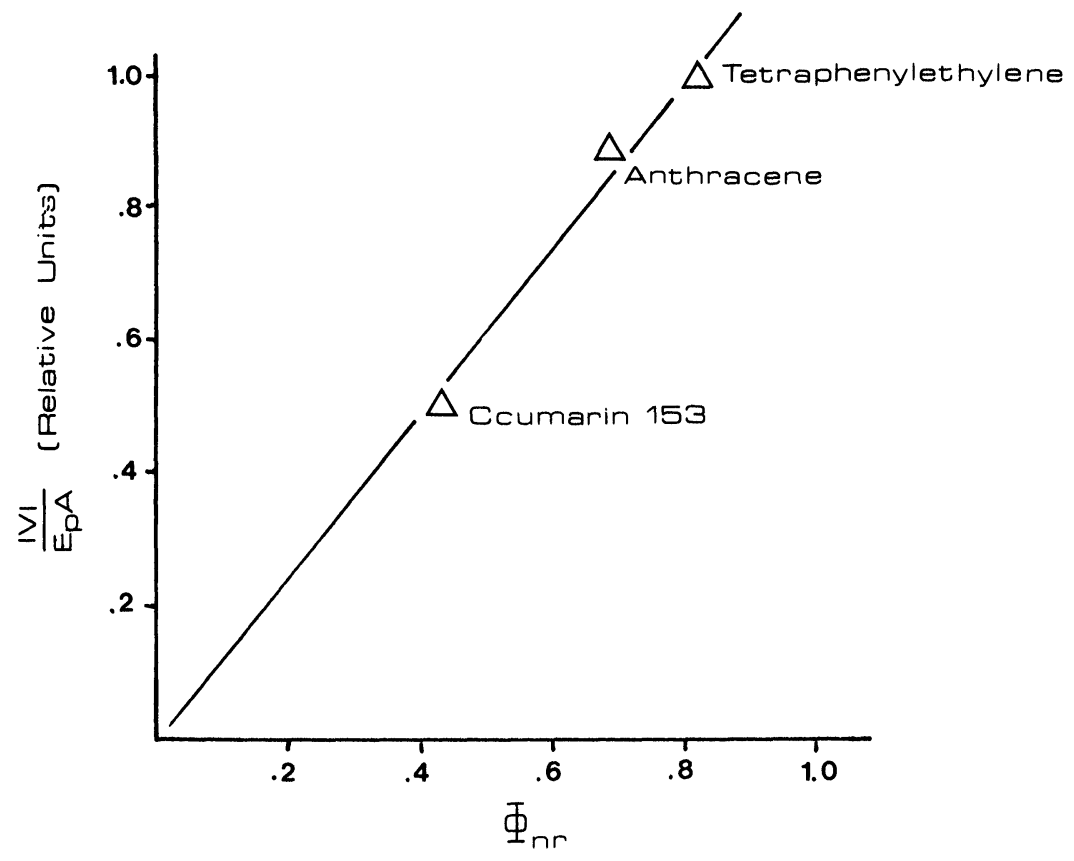

FIGURE 1 Instrumental calibration curve for cyclohexane. Each point represents calorimetric data for one compound (Table I). In order of increasing $\Phi_{n n}$, the compounds plotted are coumarin 153, anthracene, and tetraphenylethylene.

microphone, cell, experimental geometry, and amplifier gain, and must be recalculated if any of these factors is changed.

Despite its relatively fast response, the piezoelectric microphone employed here cannot resolve rapid photochemical and photophysical phenomena. Relaxation processes taking place on the picosecond and nanosecond timescales appear as impulse functions to the microphone. The $100 \mathrm{kHz}$ resonant frequency of the microphone, on the other hand, permits us to measure the photoacoustic amplitude within a few microseconds of the laser pulse; in consequence, any chemical processes which require more than a few microseconds occur long after the conclusion of the experiment and so have no effect on the observed signal.

$\mathrm{Cr}(\mathrm{CO})_{6}$ presents a particularly simple case. This compound does not fluoresce or phosphoresce. ${ }^{1}$ No excited states are detectable on 
TABLE I

Quantum yields and normalized calorimetric information on the compounds used for standardization $^{\mathrm{a}}$

\begin{tabular}{lcccc}
\hline \multicolumn{1}{c}{ Compound } & $\Phi_{f}$ & $\nu_{f}(\mathrm{WN})$ & $\Phi_{n r}$ & $\begin{array}{c}\text { Normalized } \\
|V| / E_{p} A\end{array}$ \\
\hline Tetraphenylethylene & 0.25 & 20700 & 0.82 & 1.00 \\
Anthracene & 0.36 & 24900 & 0.68 & 0.89 \\
Coumarin 153 & 0.73 & 21970 & 0.43 & 0.50 \\
\hline
\end{tabular}

a Quantum Yields and average fluorescence data are from Ref. 19.

the picosecond timescale; photofragmentation occurs in high quantum yield within 25 ps of excitation. ${ }^{2}$ The coordinatively unsaturated pentacarbonyl is notoriously reactive ${ }^{1}$ and subject to attack by even the poorest nucleophiles. Nevertheless, by using sufficiently dilute solutions and reasonably pure solvents, we can ensure that nucleophilic attack will proceed negligibly in the small number of microseconds needed to complete our experiment. Conventional calorimetric measurements ${ }^{14}$ require many seconds or minutes, and cannot in general exclude contributions from secondary reactions should they occur.

The small number of laser pulses (usually 80 pulses are averaged), brief duration of the experiment, and the rather weak fluences employed all help avoid complications arising from sample decomposition. Sample decomposition, evidenced by bleaching, accumulation of light-absorbing products, or formation of particulate matter, is readily detected by the microcomputer.

Spectrograde cyclohexane (glass distilled, Eastman Kodak) and EtOH (US Industrial Corp.) were used. $\mathrm{Cr}(\mathrm{CO})_{6}(99 \%)$ was purchased from Aldrich and used without further purification. Sample absorbances were recorded with a Perkin-Elmer Lambda III UV-Vis Spectrometer. On the basis of known extinction coefficients, the concentration of metal carbonyls ranged from $10^{-4}$ to $10^{-6} \mathrm{M}$.

\section{RESULTS AND DISCUSSION}

In Figure 2, experimentally observed photoacoustic amplitudes are plotted as a function of sample optical density for several of the 


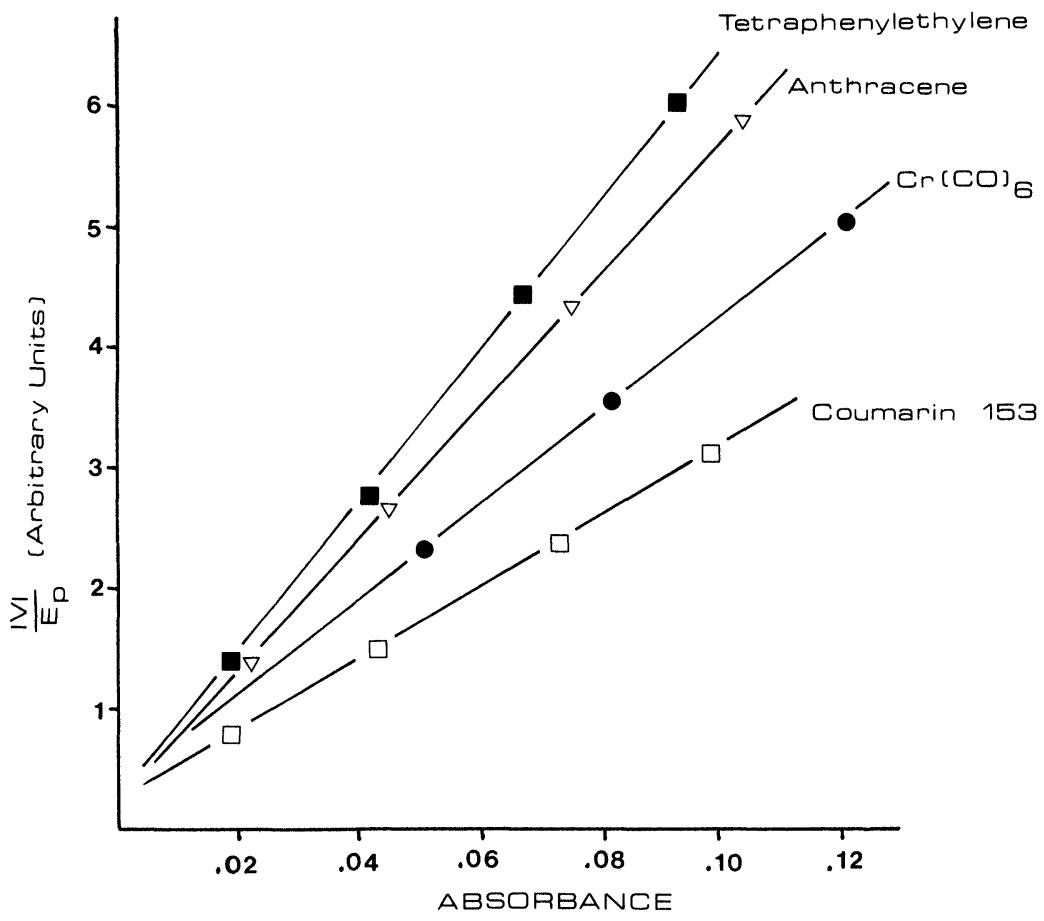

FIGURE 2 Normalized photoacoustic signal vs. Absorbance for the calibration compounds in cyclohexane and $\mathrm{Cr}(\mathrm{CO})_{6}$.

calibration compounds and $\operatorname{Cr}(\mathrm{CO})_{6}$. The calibration procedure described above relates the slope of each line in Figure 2 to a nonradiative quantum yield $\Phi_{n r}$.

In order to complete the calculation of the reaction enthalpy for cleavage of an $\mathrm{Cr}-\mathrm{CO}$ bond, we must know the following: the efficiency of the photodissociation process and the decay kinetics of the primary intermediate.

The quantum yield for the photoelimination of $\mathrm{CO}$ from $\mathrm{Cr}(\mathrm{CO})_{6}$, once generally held to be unity, has lately been the subject of considerable disagreement. In this paper, we have assumed that the quantum yield for the primary photolytic step is 1.0. A high, if not quantitative, quantum yield is suggested by extensive spectroscopic and chemical data. Theoretical investigations of the electronically excited surfaces 
of metal carbonyls suggest that the lowest excited state should be strictly dissociative. ${ }^{1,15}$ Overall chemical yields have been found to be less than unity, ${ }^{16}$ varying with different nucleophiles and experimental conditions. ${ }^{1,17}$ These results suggest that the apparent quantum yields are reduced by recombination of the reactive pentacarbonyl species with CO. However, if the initial step is strictly dissociative, the largest known chemical yield must represent a lower bound for the quantum yield of initial $\mathrm{CO}$ dissociation. If $\mathrm{CO}$ recombination is the predominant cause of nonquantitative yields, we have previously shown that this recombination process does not proceed appreciably in the period from 25 ps to $250 \mathrm{~ns}$ after photolysis. ${ }^{2}$ If it occurs on timescales longer than a few microseconds, it will not contribute to the photoacoustic signal. It remains possible, however, that recombination might occur on the subpicosecond timescale, or that some excited molecules fail to lose $\mathrm{CO}$ at all. Quantum yields ranging from 0.67 to 0.81 have recently been reported for various photosubstitution reactions. ${ }^{16,17}$

In the absence of an exact quantum yields, it is important to stress that, using the assumption of unit quantum yield, we obtain a rigorous lower bound for the metal-carbonyl bond strength. Should the actual quantum yield be less than unity, the calculated bond energy would increase proportionately.

The decay kinetics of the primary intermediate formed in the photolysis of $\mathrm{Cr}(\mathrm{CO})_{6}$ in cyclohexane have recently been studied using picosecond absorption spectroscopy. ${ }^{2}$ The primary intermediate, $\lambda_{\max }=503 \mathrm{~nm}$, was observed within 25 ps following photolysis. No change in band shape or intensity was observed for microseconds following photolysis.

With the above information, we can now use the results presented in Figures 1 and 2 to calculate the energy required to break the $\mathrm{Cr}-\mathrm{CO}$ bond and solvate the resulting fragments. This is found to be $36.9 \mathrm{kcal} / \mathrm{mol}$. Gas phase photolysis studies have measured an average bond dissociation energies for $\mathrm{Cr}(\mathrm{CO})_{6}$ of $25.8 \mathrm{kcal} / \mathrm{mol}^{4}$ Thus, for the photolysis of $\mathrm{Cr}(\mathrm{CO})_{6}$ in cyclohexane the energy needed to cleave the first $\mathrm{M}-\mathrm{CO}$ bond exceeds the average gas-phase bond dissociation energy by at least $11 \mathrm{kcal} / \mathrm{mol}$.

The observed heat of reaction is in excellent agreement with the activation enthalpies found from kinetic studies of thermal displacement reactions ${ }^{18}$ using $\mathrm{Cr}(\mathrm{CO})_{6}$. An activation energy of $40.2 \mathrm{kal} / \mathrm{mol}$ 
was determined for the formation of $\operatorname{Cr}(\mathrm{CO})_{5}$ and $\mathrm{CO}$. Thus, in comparison to the remaining $\mathrm{M}-\mathrm{CO}$ bonds, the first $\mathrm{M}-\mathrm{CO}$ bond in $\mathrm{Cr}(\mathrm{CO})_{6}$ is exceptionally difficult to cleave.

\section{Acknowledgement}

This work is supported by a grant from the National Science Foundation, CHE8117519 and the Henry and Camille Dreyfus foundation. We would like to thank Lewis Rothberg for his help in the development of photoacoustic calorimetry.

\section{References}

1. (a) G. J. Geoffroy and M. S. Wrighton, Organometallic Photochemistry (Academic Press, New York, 1979); (b) M. S. Wrighton, Chem. Rev. 74, 401 (1974).

2. (a) J. D. Simon and K. S. Peters, Chem. Phys. Let., in press; (b) J. A. Welch, K. S. Peters and V. Vaida, J. Phys. Chem. 86, 1941 (1982).

3. F. A. Cotton, A. K. Fischer and G. J. Wilkensen, J. Amer. Chem. Soc. 78, 5168 (1956).

4. J. A. Connor, Top. Curr. Chem. 71, 71 (1977).

5. M. G. Rockley and K. M. Waugh, Chem. Phys. Let. 54, 597 (1978).

6. M. J. Adams, J. G. Highfield and G. F. Kirkbright, Anal. Chem. 49, 1850 (1977).

7. I. O. Starobogatov, Opic. Spectrosc. (USSR) 42, 179 (1977).

8. W. Lahmann and H. J. Ludwig, Chem. Phys. Let. 45, 177 (1977).

9. L. J. Rothberg, J. D. Simon, M. Bernstein and K. S. Peters, J. Amer. Chem. Soc. in press.

10. Transducer Products Corporation material LTZ-2. C. K. N. Patel and A. C. Tam, Rev. Mod. Phys. 53, 115 (1981).

11. E. Voigtman, A. Jurgensenm and J. Winefordner, Anal. Chem. 53, 1442 (1981).

12. M. Bernstein, Byte 4, 465 (1982).

13. For the standard compounds the nonradiative quantum yield, $\Phi_{n r}$ is defined by the following equation:

$$
\Phi_{n r}=1-\Phi_{f}\left[\frac{\nu_{f}}{\nu_{\mathrm{exc}}}\right]
$$

where $\Phi_{f}$ is the fluorescence quantum yield, $\nu_{\text {exc }}$ is the frequency of the exciting light, and $\nu_{f}$ is the average fluorescence frequency.

14. M. Nakashima and A. W. Adamson, J. Phys. Chem. 86, 2905 (1982).

15. N. A. Beach and H. B. Gray, J. Amer. Chem. Soc. 90, 5713 (1968).

16. J. Nasielski and A. Colas, Inorg. Chem. 17, 237 (1978).

17. J. Nasielski and A. Colas, J. Organomet. Chem. 101, 215 (1975).

18. (a) R. J. Dennenberg and D. J. Darensbourg, Inorg. Chem. 11, 72 (1972).

19. (a) I. B. Berleman, Handbook of Fluorescence Spectra of Aromatic Molecules (second edition, Academic Press, New York, 1971); (b) S. Sharafy and K. A. Muszkat, J. Amer. Chem. Soc. 93, 4119 (1971); (c) G. Jones, W. R. Jackson and A. Halpern, Chem. Phys. Lett. 72, 391 (1980). 